\title{
Soothing and anti-itch effect of quercetin phytosome in human subjects: a single-blind study
}

This article was published in the following Dove Press journal:

Clinical, Cosmetic and Investigational Dermatology

26 February 2016

Number of times this article has been viewed

\section{Giada Maramaldi' \\ Stefano Togni' \\ Ivan Pagin' \\ Luca Giacomelli² \\ Roberta Cattaneo 3 \\ Roberto Eggenhöffner ${ }^{2}$ \\ Samuele E Burastero ${ }^{4}$ \\ 'Indena S.p.A, Milan, ${ }^{2}$ Department of Surgical Sciences and Integrated Diagnostics, School of Medicine, Genova University, Genoa, ${ }^{3}$ Abich Srl, Verbania, ${ }^{4}$ San Raffaele Scientific Institute, Milan, Italy}

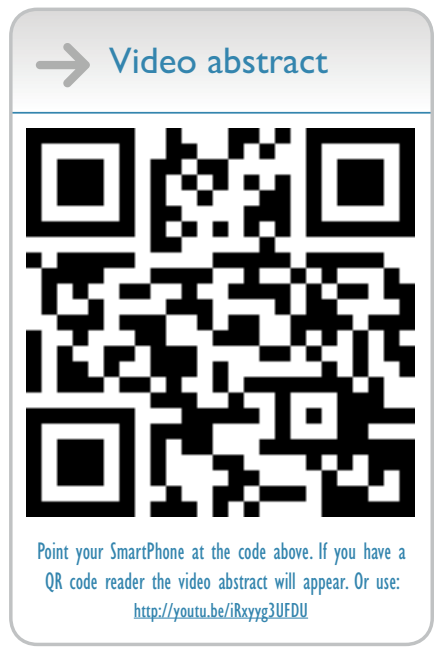

Correspondence: Giada Maramaldi Indena S.p.A.,Viale Ortles I2, Milan, 20139 Italy

Tel +390257496249

Fax +390257496374

Email giada.maramaldi@indena.com
Background: We evaluated the ability of quercetin, a natural antioxidant formulated in a specific delivery system, to reduce skin inflammation induced by a variety of stimuli, including UV radiation, stimulation with a histamine solution, or contact with chemical irritants. In particular, we tested the soothing and anti-itch effect of Quercevita ${ }^{\circledR}, 1 \%$ cream for external use, a formulation characterized by a phospholipids-based delivery system.

Patients and methods: The study was a monocentric, single blind trial that enrolled a group of 30 healthy volunteers. The back of each subject was examined to identify four quadrants with no previous skin damage or naevi that were treated in order to induce a controlled and reversible form of skin stress. The areas were treated as follows: no product; Quercevita ${ }^{\circledR} 1 \%$ cream, $2 \mathrm{mg} / \mathrm{cm}^{2}$; placebo; positive control (a commercially available topical formulation containing $1 \%$ dexchlorpheniramine).

Results: Only quercetin phospholipids $1 \%$ and dexchlorpheniramine $1 \%$ achieved a significant reduction in erythema with comparable results: $(-10.05 \%[P=0.00329]$ for quercetin phospholipids $1 \%$ vs $-14.05 \%$ [ $P=0.00046]$ for the positive control). Moreover, quercetin phospholipids $1 \%$ and dexchlorpheniramine $1 \%$ were both associated with a significant decrease in mean wheal diameter: ( $-13.25 \%$ and $-12.23 \%$ for dexchlorpheniramine $1 \%$, respectively). Similar findings were reported for the other tested parameters.

Conclusion: Quercetin has a skin protective effect against damage caused by a variety of insults, including UV radiation, histamine, or contact with toxic chemical compounds. Indeed, quercetin is able to reduce redness, itching, and inflammation of damaged skin; it may also help restore skin barrier function, increasing hydration, and reducing water loss.

Keywords: quercetin, anti-oxidant effect, anti-inflammation, reactive oxygen species

\section{Introduction}

The skin is subjected to continuous insults from environmental factors, external trauma, or pathological conditions. ${ }^{1}$ The production of reactive oxygen species (ROS), which results from exposure to UV radiation or contact with various toxic chemicals, is one of the main causes of skin damage and may contribute to the development of skin inflammation disorders, skin cancer, and cutaneous photoaging. ${ }^{2,3}$

Although the skin presents different mechanisms to protect itself from damage caused by ROS, the capacity of the system is limited and cannot cope with excessive exposure to oxidative damage. The use of topically applied antioxidants has been proposed to support the cutaneous antioxidant defense systems, thanks to their ability to quench free radicals and stop the inflammation process. ${ }^{3-6}$ Among the various compounds investigated for their anti-oxidant properties, flavonoids have emerged 
for their remarkable anti-oxidant, anti-inflammatory, chemo-protective, and chemo-therapeutic effects. ${ }^{7-9}$

Quercetin is one of the most abundant natural flavonoids, commonly found in fruits and vegetables. ${ }^{10}$ It shows superior anti-oxidant activity within the flavonoids family ${ }^{11}$ and it acts mainly by scavenging oxygen radicals, ${ }^{12}$ protecting lipids against peroxidation, and chelating metal ions. ${ }^{13}$ In addition, it displays anti-inflammatory properties, including inhibition of cytokine production such as TNF- $\alpha^{14}$ and IL-8. ${ }^{15}$ The results of some animal studies have also reported its anti-nociceptive (pain-relieving) effects that might help reduce inflammatory-related pain. ${ }^{11,16}$

Despite the promising beneficial properties attributed to quercetin, it is important to underline that most of the information is derived from in vitro or in vivo animal studies, while the experience of the skin protective effect of quercetin in human subjects is still limited.

One of the main limits to the use of quercetin as skin protecting agent is its poor water solubility and low skin permeability; ${ }^{17}$ in fact, suitable percutaneous absorption is considered an essential requirement for topical protective agents. Many researchers have focused on the development of new strategies to increase quercetin skin permeability, including the use of prodrugs, liposomes, and novel vesicular systems. ${ }^{18-20}$

In this study we evaluated the ability of quercetin (formulated in a specific delivery system) to reduce skin inflammation induced by a variety of stimuli, including UV radiation, stimulation with a histamine solution, or contact with chemical barrier distributors such as sodium lauryl sulfate (SLS) or glycolic acid (GA).

In particular we tested the soothing and anti-itch effect of Quercevita ${ }^{\circledR}, 1 \%$ cream for external use, a formulation characterized by a phospholipids-based delivery system used to increase the ability of quercetin to permeate the skin.

\section{Patients and methods}

\section{Study design}

This study was conducted in line with the Declaration of Helsinki and was approved by the independent ethics committee ALLTOX IEC (ref 661-001-00260115).

The study was a monocentric, single blind trial that enrolled a group of 30 healthy volunteers. The back of each subject was examined to identify four quadrants with no previous skin damage or naevi that were treated in order to induce a controlled and reversible form of skin stress. Each identified quadrant was divided into four areas and treated as follows:
- Quadrant 1: exposure to a fixed dose of UVA + UVB radiations emitted by a solar simulator, equal to $150 \%$ of the minimal erythema-inducing dose; the dose was calculated on the basis of subject's specific phototype.

- Quadrant 2: prick test with histamine. This procedure was performed on the back and also on the volar surface of the forearm of each volunteer in order to obtain a more precise itch rating.

- Quadrant 3: application of four patches containing $0.07-0.1 \mathrm{~mL}$ of SLS solution $1 \%$; each patch was left in contact with the skin for 24 hours.

- Quadrant 4: application of four patches containing $0.07-0.1 \mathrm{~mL}$ of GA solution $1 \%$; each patch was left in contact with the skin for 24 hours.

The four identified areas of each quadrant were than treated with the following products, applied by specialized personnel in non-occlusive cutaneous applications:

- Negative control: no product.

- Active treatment: Quercevita ${ }^{\circledR} 1 \%$ cream, $2 \mathrm{mg} / \mathrm{cm}^{2}$; sunflower phospholipids-based delivery system of quercetin, according to the Phytosome ${ }^{\circledR}$ technology. The exact composition is reported in Table 1.

- Placebo: placebo formulation (same composition as the tested formulation but devoid of the $1 \%$ quercetin, replaced by water), $2 \mathrm{mg} / \mathrm{cm}^{2}$.

- Positive control: a commercially available topical formulation containing 1\% dexchlorpheniramine (Polaramin ${ }^{\circledR}$, MSD, Rome, Italy) $2 \mathrm{mg} / \mathrm{cm}^{2}$.

The following parameters were assessed after the induction of skin inflammation (T0) and following the application of the products, at defined time-points:

- Quadrant 1: erythema(E), measured with the Mexameter $^{\circledR}$ index at T0 (24 hours after irradiation) and T4 (4 hours after treatment application).

Table I Qualitative and quantitative composition of Quercevita ${ }^{\circledR}$ I\% cream

\begin{tabular}{ll}
\hline Ingredient & $\%$ \\
\hline Water & 82.40 \\
Glycerin & 2.00 \\
Disodium EDTA & 0.10 \\
Polyacrylamide, CI3-I4 Isoparaffin, Laureth 7 & 3.00 \\
Hydrogenated polydecene & 8.50 \\
Caprylic/capric triglycerides & 2.00 \\
Lecithin (synonym: phosphatidylcholine), quercetin & 1.00 \\
Lecithin, tocopherol, ascorbyl palmitate, citric acid & 0.10 \\
Phenoxyethanol & 0.60 \\
Imidazolidinyl urea & 0.30 \\
\hline
\end{tabular}

Abbreviation: EDTA, ethylenediaminetetraacetic acid. 
- Quadrant 2: wheal diameter ( $\mathrm{mm}$ ) and subjective evaluation of itching sensation measured with the visual analog scale (VAS; from $0=$ no itch to $10=$ maximum itch) at $\mathrm{T} 0$ (10 minutes after prick test), T10m, and T30m (10 and 30 minutes after treatment application).

- Quadrant 3 and 4: E and hydration value at T0 (15 minutes after removal of the patches), T2, and T4 (2 and 4 hours after treatment application); trans-epidermal water loss (TEWL) at T0 (15 minutes after removal of the patches), T4, and T24 (4 and 24 hours after treatments application).

An empty patch was applied on the back of each volunteer and left in contact with the skin for 24 hours as a negative control for erythema. In this area $\mathrm{E}$ was measured 15 minutes after patch removal.

\section{Population}

The healthy volunteers included in the study were identified from the database of volunteers of the Abich Test Center and were adult (from 18 to 65 years old) male or female subjects. All the participants were able to understand the characteristics of the study, the requested observation times, the tasks implied, and the possible risks, and they all signed an informed consent form before the enrollment.

The following exclusion criteria were applied:

- pregnancy or nursing condition;

- blemishes, marks, including tattoos, scars, sunburn on the test site(s), or any skin lesions which might interfere with the test evaluation;

- medication (local and/or systemic) which might interfere with the test evaluation;

- participation in other simultaneous studies that might interfere with the test evaluation or participation in a previous one;

- allergy to histamine.

\section{Statistical analysis}

Continuous variables were analyzed by descriptive statistics. The Student's $t$-test analysis was performed to evaluate the significance of the differences measured between different time-points; a $P$-value $<0.05$ was considered statistically significant.

\section{Results \\ UV irradiation}

UV irradiation resulted in a similar development of erythema in the four treated areas. The average $\mathrm{E}$ values measured before (T0) and after (T4) treatment application are reported in Table 2, together with the percentage variations between the two time-points. Overall, only quercetin phospholipids $1 \%$ and dexchlorpheniramine $1 \%$ achieved a significant reduction in erythema with comparable results: $-10.05 \%(P=0.00329)$ for quercetin phospholipids $1 \%$ vs $-14.05 \%(P=0.00046)$ for the positive control. Both placebo and no treatment resulted in a non-significant decrease in erythema (Table 2).

\section{Histamine prick test}

After the histamine prick test the mean wheal diameter was similar in the four affected areas. None of the products were able to reduce the mean wheal diameter 10 minutes after the application. However, after 20 additional minutes, quercetin phospholipids $1 \%$ and dexchlorpheniramine $1 \%$ were both associated with a significant decrease in mean wheal diameter: $-13.25 \%$ for quercetin phospholipids $1 \%$ (from 4.2 to $3.6 \mathrm{~mm} ; P=0.00019)$ and $-12.23 \%$ for dexchlorpheniramine $1 \%$ (from 4.0 to $3.5 ; P=0.00257$ ). At T30, the mean wheal diameter was also slightly reduced with no treatment or when the placebo formulation was applied; however this reduction was extremely limited and not statistically significant (Figure 1).

According to the results of the VAS test, subjects experienced a reduction in itching with all the treatments; the decrease in the itching sensation started 10 minutes after the application of the products, and further decreased at $\mathrm{T} 30 \mathrm{~m}$. Although the changes in the VAS score were statistically significant for all the four treatments considered - both comparing T10m vs T0 or T30m vs T10m - it must be noted that after 10 minutes quercetin phospholipids $1 \%$ and dexchlorpheniramine $1 \%$ achieved a higher reduction in VAS score compared to placebo or no treatment (Figure 2).

\section{SLS solution}

The erythema values measured after the application of SLS patches and after 2 and 4 hours' skin treatment are reported in Table 3. Overall, only quercetin phospholipids $1 \%$ and dexchlorpheniramine $1 \%$ were able to reduce erythema, while no

Table 2 Mean \pm standard error erythema $(E)$ values after UV irradiation (T0) and 4 hours (T4) after treatment application

\begin{tabular}{lllll}
\hline Treatment & E value T0 E value T4 \% Variations P-value \\
& & \multicolumn{3}{c}{ T0 vs T4 } \\
\hline $\begin{array}{l}\text { No treatment } \\
\text { Quercetin } \\
\text { phospholipids I\% }\end{array}$ & $313 \pm 15$ & $310 \pm 14$ & $0.68 \%$ & 0.389 \\
$\begin{array}{l}\text { Placebo } \\
\begin{array}{l}\text { Dexchlorpheniramine } \\
\text { I\% }\end{array}\end{array}$ & $312 \pm 12 \pm 14$ & $273 \pm 14$ & $-10.05 \%$ & 0.003 \\
\hline
\end{tabular}




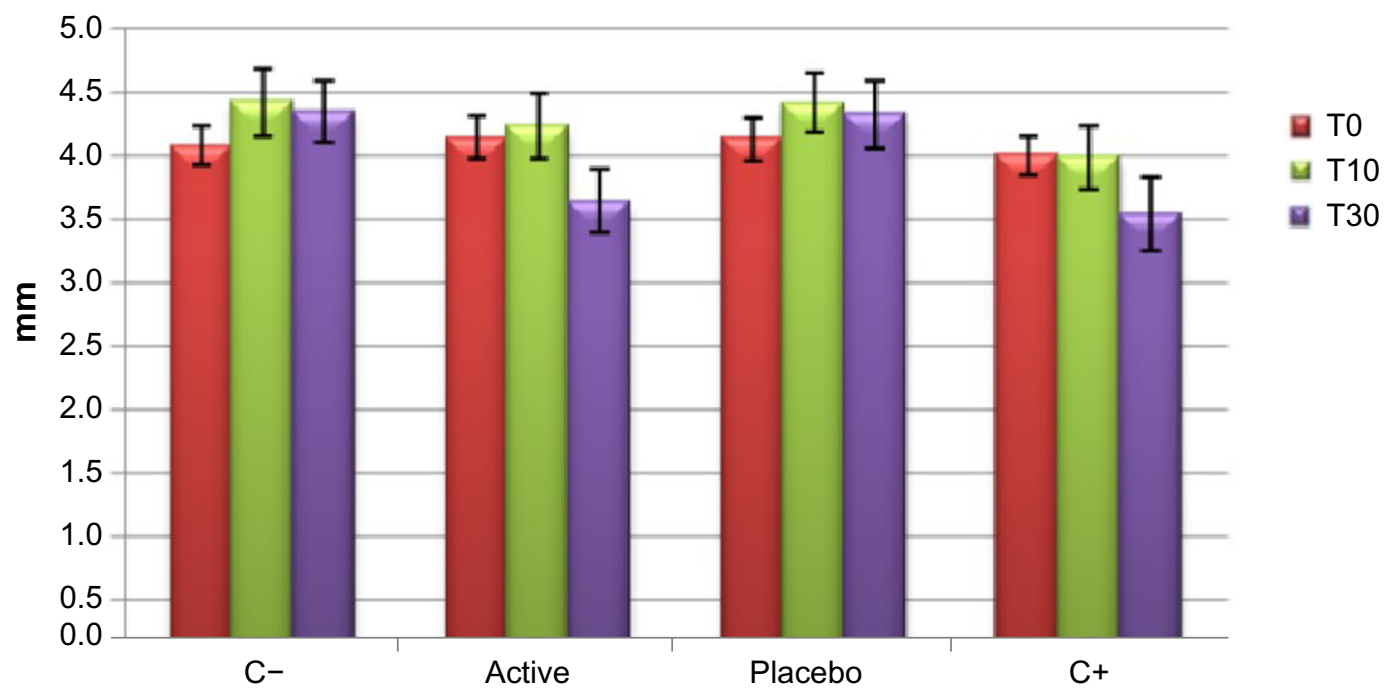

Figure I Mean wheal diameter after histamine prick test (T0) and 10 (TI0) and 30 (T30) minutes after treatment application. Notes: Bars represent standard deviation. $\mathrm{C}-$ is the non-treated (or control area). $\mathrm{C}+$ is the positive control area.

significant effect was reported for placebo and no treatment. The reduction in $\mathrm{E}$ values was already visible 2 hours after quercetin phospholipids $1 \%(-7.30 \% ; P=0.00027)$ and dexchlorpheniramine $1 \%(-9.50 \% ; P=0.00397)$ application and was even more visible after 4 hours: $-17.01 \%(P<0.0001)$ for quercetin phospholipids $1 \%$ and $-18.39 \%(P<0.0001)$ for dexchlorpheniramine $1 \%$ (Table 3).

The hydration values were increased both after 2 and 4 hours in the areas treated with quercetin phospholipids $1 \%$ and dexchlorpheniramine $1 \%$. Interestingly, after 2 hours of treatment only quercetin phospholipids $1 \%$ was able to induce a significant increase in hydration $(12.85 \% ; P=0.025)$. A positive trend was observed for dexchlorpheniramine $1 \%$ $(10.73 \%)$, but the difference was not statistically signifi- cant $(P=0.29761)$. Both treatments reported a statistically significant increase in hydration at T4: $24.03 \%(P<0.0001)$ for quercetin phospholipids $1 \%$, and $19.23 \%(P=0.04702)$ for dexchlorpheniramine $1 \%$. While placebo had no effect on the hydration level, the absence of treatment resulted in a slight but statistically significant decrease in hydration at T4 (-3.16\%, $P=0.04705)$.

The mean TEWL values were significantly decreased in areas treated with quercetin phospholipids $1 \%$ and dexchlorpheniramine $1 \%$ both after 4 and 24 hours' treatment. At T4 the mean decrease in TEWL was $-24.94 \%(P=0.00015)$ for quercetin phospholipids $1 \%$ and $-24.00 \%$ ( $P=0.00069)$ for dexchlorpheniramine 1\%; after 24 hours the registered values were $-38.10 \%(P<0.0001)$ and $-35.86 \%(P<0.0001)$,

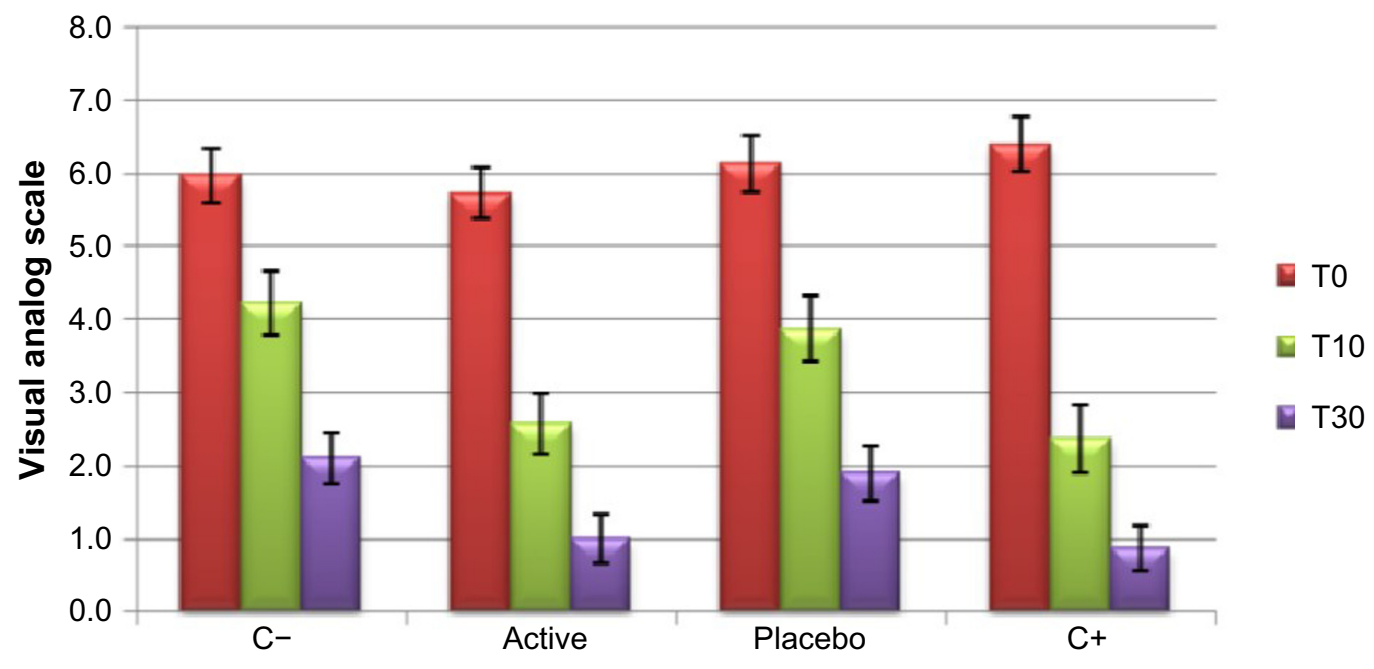

Figure 2 Mean itching values after histamine prick test (T0) and 10 (TI0) and 30 (T30) minutes after treatment application. Notes: Bars represent standard deviation. $\mathrm{C}-$ is the non-treated (or control area). $\mathrm{C}+$ is the positive control area. 
Table 3 Mean \pm standard error erythema values after application of SLS patches (T0) and 2 (T2) and 4 (T4) hours after treatment application

\begin{tabular}{|c|c|c|c|c|c|}
\hline Treatment & TO & T2 & T4 & $\begin{array}{l}\% \text { Variations T2 vs T0 } \\
\text { (P-value) }\end{array}$ & $\begin{array}{l}\text { \% Variations T4 vs T0 } \\
\text { (P-value) }\end{array}$ \\
\hline No treatment & $330 \pm 17$ & $34 I \pm 15$ & $334 \pm 15$ & $5.76 \%(P=0.11808)$ & $4.74 \%(P=0.38525)$ \\
\hline Quercetin phospholipids I\% & $351 \pm 18$ & $321 \pm 16$ & $287 \pm 15$ & $-7.30 \%(P=0.00027)$ & $-17.01 \%(P<0.0001)$ \\
\hline Placebo & $321 \pm 17$ & $331 \pm 18$ & $319 \pm 16$ & $4.87 \%(P=0.13174)$ & $1.43 \%(P=0.41323)$ \\
\hline Dexchlorpheniramine $1 \%$ & $340 \pm 16$ & $307 \pm 19$ & $274 \pm 17$ & $-9.50 \%(P=0.00397)$ & $-18.39 \%(P<0.0001)$ \\
\hline
\end{tabular}

Abbreviation: SLS, sodium lauryl sulfate.

for the two treatments, respectively. Of note, the absence of treatment also resulted in a significant decrease in TEWL values, although the reduction was considerably less than that obtained with the active treatment or the positive control $(-11.01 \%, P=0.02046)$. No remarkable difference was observed with placebo.

\section{GA solution}

The results observed after treatment with GA were similar to those reported with the SLS solution. Only quercetin phospholipids $1 \%$ and dexchlorpheniramine $1 \%$ resulted in a significant decrease in $\mathrm{E}$ values both after 2 hours $(-8.20 \%$, $P=0.00064$ and $-8.68 \%, P<0.0001$, respectively) and 4 hours $(-16.83 \%, P<0.0001$ and $-17.80 \%, P<0.0001$, respectively). No statistically significant difference was reported with placebo or no treatment (Table 4).

A positive trend toward the increase in hydration was measured at $\mathrm{T} 2$ and $\mathrm{T} 4$ with quercetin phospholipids $1 \%$ and dexchlorpheniramine $1 \%: 8.30 \%$ and $16.48 \%$ with quercetin phospholipids $1 \%$, and $6.29 \%$ and $9.29 \%$ with dexchlorpheniramine $1 \%$, at the two time-points, respectively. However, the increase was statistically significant only after 4 hours of quercetin phospholipids $1 \%$ treatment: $16.48 \%(P=0.00863)$. Both placebo and no treatment resulted in a continuous but not statistically significant reduction in hydration.

TEWL was significantly decreased after 4 and 24 hours in areas treated with quercetin phospholipids $1 \%$ and dexchlorpheniramine $1 \%$. The percentage variation measured at T4 was $-32.15 \%(P=0.00007)$ for quercetin phospholipids $1 \%$ and $-35.37 \%(P=0.00028)$ for dexchlorpheniramine $1 \%$, while after 24 hours the reduction was equal to $-45.69 \%$ $(P<0.0001)$ and $-54.28 \%(P=0.00001)$, respectively, compared to T0. No significant changes were observed in areas treated with placebo, while the absence of treatment resulted in a statistically significant reduction in TEWL after 24 hours; however, the results were remarkably lower than those observed with active treatment or positive control $(-6.48 ; P=0.03707)$.

\section{Discussion}

The results of our study suggest that quercetin phospholipids $1 \%$, cream for external use, has a significant soothing effect against skin inflammation induced by different insults, including UV radiation, histamine prick test, skin barrier disruption induced by contact with SLS or GA solutions. The soothing effect is visible as a reduction in redness (erythema), wheal diameter, and itching sensation. In addition, our results suggest the ability of this formulation to help restore the skin barrier functions, given the increase in hydration and the reduction in TEWL. The separate effect of the different components - quercetin, phospholipids, and well-known antioxidants such as ascorbic acid and tocopherol - was not evaluated in this study, since they are synergic in constituting a single active entity.

The reduction in erythema obtained with quercetin phospholipids $1 \%$ was similar to that observed after antihistaminic treatment (dexchlorpheniramine 1\%), used as positive control. Indeed, 4 hours after the application, both treatments were able to achieve a significant reduction in skin redness with comparable values, regardless of the type

Table 4 Mean \pm standard error erythema values after application of GA patches (T0) and 2 (T2) and 4 (T4) hours after treatment application

\begin{tabular}{|c|c|c|c|c|c|}
\hline Treatment & T0 & T2 & T4 & $\begin{array}{l}\text { \% Variations T2 vs T0 } \\
\text { (P-value) }\end{array}$ & $\begin{array}{l}\text { \% Variations T4 vs T0 } \\
\text { (P-value) }\end{array}$ \\
\hline No treatment & $32 I \pm 17$ & $326 \pm 16$ & $322 \pm 15$ & $3.52 \%(P=0.11808)$ & $2.90 \%(P=0.46091)$ \\
\hline Quercetin phospholipids I\% & $362 \pm 17$ & $328 \pm 14$ & $296 \pm 14$ & $-8.20 \%(P=0.00064)$ & $-16.83 \%(P<0.0001)$ \\
\hline Placebo & $345 \pm 19$ & $354 \pm 18$ & $346 \pm 17$ & $5.70 \%(P=0.21274)$ & $3.23 \%(P=0.45389)$ \\
\hline Dexchlorpheniramine I\% & $359 \pm 12$ & $327 \pm 12$ & $295 \pm 14$ & $-8.68 \%(P<0.000 I)$ & $-17.80 \%(P<0.000 \mid)$ \\
\hline
\end{tabular}

Abbreviation: GA, glycolic acid. 
of agent used as skin irritant. Of note, the highest reduction in erythema was achieved for both treatments after skin exposure to GA, while the lowest effect was registered after UV radiation. The percentage variations in erythema reported for quercetin phospholipids $1 \%$ and dexchlorpheniramine $1 \%$, respectively, were: $-10.05 \%$ and $-14.05 \%$ after exposure to UV radiation, $-17.01 \%$ and $-18.39 \%$ after contact with SLS solution, and $-16.83 \%$ and $-17.80 \%$ after contact with GA solution.

The photoprotective properties of quercetin have been investigated in a number of studies. ${ }^{21,22}$ Erden Inal et al ${ }^{21}$ examined the effect of quercetin on UVA radiation on the skin in an in vivo animal study. They observed that exposure to UVA radiation resulted in oxidative damage to the skin, measured as an increase in the levels of malondialdehyde - an end-product of lipid peroxidation - and a significant decrease in antioxidant enzymes such as glutathione peroxidase, glutathione reductase, catalase, and superoxide dismutase. In rats treated with quercetin, malondialdehyde levels were significantly decreased if compared to rats that received UVA radiation alone, and the reduction in antioxidant enzymes was considerably lower. The authors concluded that treatment with quercetin might have a beneficial effect on skin cells, by protecting them or reducing the harmful effects of UVA irradiation.

In another study, conducted by Vicentini et al on the skin of hairless mice, quercetin significantly reduced skin damage caused by UVB radiation. The authors observed that daily application of a water/oil microemulsion of quercetin prevented the UVB irradiation-induced depletion of glutathione, one of the most important endogenous mechanisms for protection against UV-induced ROS. In addition, quercetin was able to reduce the secretion/activation of MMPs. These proteinases, whose levels are increased after UV radiation, act by degrading skin collagen and elastin, thus contributing to skin photoaging. ${ }^{22}$

The results of our study further confirm those previously reported on the anti-oxidant properties of quercetin and on its ability to reduce the oxidative damage caused by UV radiation. However, to our knowledge, this is the first time that the effect of quercetin on the prevention of UV-induced skin damage has been demonstrated in human subjects.

In our study we also observed that quercetin phospholipids $1 \%$ was able to reduce skin irritation caused by chemical agents such as SLS. These results are in contrast with those previously reported by Katsarou et al. ${ }^{23}$ The authors evaluated the effect of quercetin on acute skin irritation induced by SLS $2 \%$ solution in 15 patients with contact dermatitis. The results of the study showed that acute inflammation induced by SLS did not significantly improve after treatment with quercetin $7.5 \%$ and that erythema and TEWL indexes, after a consistent initial increase, were only slightly reduced after 5 days of quercetin treatment and did not return to baseline levels even after 7 days of therapy. ${ }^{23}$

Conversely, we reported not only a reduction in erythema, but also an improvement in the skin barrier functions, as revealed by the increase in hydration and the decrease in TEWL. Our results suggest that the anti-oxidant activity of quercetin, and in particular the reduction in lipid peroxidation, may have a crucial effect in the restoration of the barrier function of damaged skin. Indeed, lipids in the stratum corneum play an important role for the maintenance of the permeability barrier of the skin, and lipid peroxidation products are known for their toxic effect on mammalian skin cells. ${ }^{24}$

The differences observed between our and Katsarou et al's study may lay in the different concentrations of the agents used (SLS 1\% vs 2\%) or in the fact that, although the concentration of quercetin used by Katsarou et al was higher than that utilized in our study, the lack of an appropriate delivery system might have hampered the skin permeability of the molecule. Moreover, the different experimental and evaluation protocols followed have largely contributed to the observed discrepancy between our results and theirs.

The results observed after contact with GA solution are similar to those reported for SLS. To our knowledge no previous studies have investigated the activity of quercetin on skin irritation induced by this chemical agent.

Lastly, in our study quercetin phospholipids $1 \%$ displayed anti-histaminic properties, with a reduction in mean wheal diameter and itching after histamine prick test comparable to that achieved by the positive control (dexchlorpheniramine maleate). The anti-allergic effect of quercetin has been suggested by a number of in vitro and animal studies that showed its inhibitory action on the secretory response of activated mast cells ${ }^{25,26}$ and on the release of histamine from basophils. ${ }^{27,28}$

Of note, we conducted a separate in vitro study to assess the activity of Quercevita ${ }^{\circledR}$ in inhibiting basophil degranulation in rodent cells. Degranulation was stimulated with a cross-linking of the IgE human receptor (FcRI) with a specific polyclonal antibody and was assessed by measuring the release of $\beta$-hexosaminidase - a mediator secreted after basophils' activation. The inhibitory activity of Quercevita ${ }^{\circledR}$ was compared to that obtained with hyaluronic acid (positive control). Quercevita ${ }^{\circledR}$ resulted in an effective and dosedependent inhibition of basophil degranulation, up to a level 
of $83.6 \%$ (at $10 \mu \mathrm{g} / \mathrm{mL}$ ), compared to $79.2 \%$ obtained with the positive control. ${ }^{29}$

These results suggest that quercetin exerts a dual anti-allergy action: on one hand it inhibits the release of histamine from basophil cells; on the other, if histamine is present, it reduces skin redness and itching caused by histamine. These activities, combined with the anti-inflammatory action exerted by quercetin and the inhibition of cytokine production, might account for the reduction in skin irritation observed in our study. These results prompt future study to investigate the skin protective effect of quercetin on skin damage related to allergic reactions. In addition, it would be useful to explore other potential delivery methods (eg, nanoparticles, pending a proper and careful assessment of their safety in line with European Union requirements).

\section{Conclusion}

Overall the results of our study suggest that Quercevita ${ }^{\circledR}$ $1 \%$ has a skin protective effect against damage caused by a variety of insults, including UV radiation, histamine, or contact with toxic chemical compounds. Indeed, this formulation is able to reduce redness, itching, and inflammation of damaged skin; in addition it may help restore skin barrier function, increasing hydration, and reducing water loss. These effects are achieved mainly due to the anti-oxidant and antiinflammatory properties of quercetin, enabling it to scavenge free radicals and reduce the oxidative damage caused by UV radiation, decrease lipid peroxidation, and inhibit the production of inflammatory enzymes and other cytokines.

Moreover, quercetin exhibits anti-allergic properties, as it reduces irritation and itching caused by histamine prick test. This activity adds to the inhibitory effect exerted by quercetin on basophil degranulation and consequential histamine release.

To our knowledge, this is the first study where the multiple properties of quercetin on skin soothing and protection have been reported in human subjects.

\section{Acknowledgment}

Editorial assistance was provided by Ambra Corti; this assistance was supported by internal funds.

\section{Disclosure}

GM, ST, and IP are employees of Indena S.p.A; LG is a consultant of Indena S.p.A. The other authors report no conflicts of interest in this work.

\section{References}

1. Saija A, Tomaino D, Trombetta M, Giacchi A, De Pasquale F, Bonina F. Influence of different penetration enhancers on in vitro skin permeation and in vivo photoprotective effect of flavonoids. Int $J$ Pharm. 1998;175(1):85-94.
2. Shindo Y, Witt E, Packer L. Antioxidant defense mechanisms in murine epidermis and dermis and their responses to ultraviolet light. $J$ Invest Dermatol. 1993;100(3): 260-265.

3. Wang ZY, Huang MT, Ferraro T, et al. Inhibitory effect of green tea in the drinking water on tumorigenesis by ultraviolet light and 12-Otetradecanoylphorbo13-acetate in the skin of SKH-1 mice. Cancer Res. 1992;52(5):1162-1170.

4. Phan TT, See P, Lee ST, Chan SY. Protective effects of curcumin against oxidative damage on skin cells in vitro: its implication for wound healing. J Trauma. 2001;51(5):927-931.

5. Shetty S, Udupa S, Udupa L. Evaluation of antioxidant and wound healing effects of alcoholic and aqueous extract of ocimum sanctum linn in rats. Evid Based Complement Alternat Med. 2008;5(1): 95-101.

6. Akkol EK, Süntar I, Orhan IE, Keles H, Kan A, Coksari G. Assessment of dermal wound healing and in vitro antioxidant properties of Avena sativa. J Cereal Sci. 2011;53(3):285-290.

7. Svobodová A, Psotová J, Walterová D. Natural phenolics in the prevention of UV-induced skin damage. A review. Biomed Pap Med Fac Univ Palacky Olomouc Czech Repub. 2003;147(2):137-145.

8. Pinnell SR. Cutaneous photodamage, oxidative stress, and topical antioxidant protection. J Am Acad Dermatol. 2003;48(1):1-22.

9. Nayak BS, Ramdath DD, Marshall JR, et al. Wound-healing activity of the skin of the common grape (Vitis Vinifera) variant, Cabernet Sauvignon. Phytother Res. 2010;24(8):1151-1157.

10. Kelly GS. Quercetin. Monograph. Altern Med Rev. 2011;16(2): 172-194.

11. Valério DA, Georgetti SR, Magro DA, et al. Quercetin reduces inflammatory pain: inhibition of oxidative stress and cytokine production. J Nat Prod. 2009;72(11):1975-1979.

12. Cushnie TP, Lamb AJ. Antimicrobial activity of flavonoids. Int J Antimicrob Agents. 2005;26(5):343-356.

13. Wagner C, Vargas AP, Roos DH, et al. Comparative study of quercetin and its two glycoside derivatives quercetin and rutin against methylmercury (MgHg)-induced ROS production in rat brain slices. Arch Toxicol. 2010;84(2):89-97.

14. Chuang CC, Martinez K, Xie G, et al. Quercetin is equally or more effective than resveratrol in attenuating tumor necrosis factor- $\{$ alpha $\}$ mediated inflammation and insulin resistance in primary human adipocytes. Am J Clin Nutr. 2010;92(6):1511-1521.

15. Geraets L, Moonen HJ, Brauers K, Wouters EF, Bast A, Hageman GJ. Dietary flavones and flavonoles are inhibitors of poly(ADP-ribose)polymerase-1 in pulmonary epithelial cells. J Nutr. 2007;137(10):2190-2195.

16. Filho AW, Filho VC, Olinger L, de Souza MM. Quercetin: further investigation of its antinociceptive properties and mechanisms of action. Arch Pharm Res. 2008;31(6):713-721.

17. Bose S, Du Y, Takhistov P, Michniak-Kohn B. Formulation optimization and topical delivery of quercetin from solid lipid based nanosystems. Int J Pharm. 2013;441(1-2):56-66.

18. Montenegro L, Carbone $\mathrm{C}$, Maniscalco $\mathrm{C}$, et al. In vitro evaluation of quercetin-3-O-acyl esters as topical prodrugs. Int J Pharm. 2007;336(2):257-262.

19. Chessa M, Caddeo C, Valenti D, Manconi M, Sinico C, Fadda AM. Effect of penetration enhancer containing vesicles on the percutaneous delivery of quercetin through new born pig skin. Pharmaceutics. 2011;3(3):497-509.

20. Tan Q, Liu W, Guo C, Zhai G. Preparation and evaluation of quercetinloaded lecithin-chitosan nanoparticles for topical delivery. Int J Nanomedicine. 2011;6:1621-1630.

21. Erden Inal M, Kahraman A, Köken T. Beneficial effects of quercetin on oxidative stress induced by ultraviolet A. Clin Exp Dermatol. 2001;26(6):536-539.

22. Vicentini FT, Simi TR, Del Ciampo JO, et al. Quercetin in w/o microemulsion: in vitro and in vivo skin penetration and efficacy against UVB-induced skin damages evaluated in vivo. Eur J Pharm Biopharm. 2008;69(3):948-957. 
23. Katsarou A, Davoy E, Xenos K, Armenaka M, Theoharides TC. Effect of an antioxidant (quercetin) on sodium-lauryl-sulfate-induced skin irritation. Contact Dermatitis. 2000;42(2):85-89.

24. Yagi K. Lipid peroxides in the skin. In: Hayaishi O, Imamura S, Miyachi Y, editors. The biological role of reactive oxygen species in the skin. New York: Elsevier; 1987:109-116.

25. Min YD, Choi CH, Bark H, et al. Quercetin inhibits expression of inflammatory cytokines through attenuation of NF-kappaB and p38 MAPK in HMC-1 human mast cell line. Inflamm Res. 2007;56(5):210-215.

26. Kempuraj D, Castellani ML, Petrarca C, et al. Inhibitory effect of quercetin on tryptase and interleukin- 6 release, and histidine decarboxylase mRNA transcription by human mast cell-1 cell line. Clin Exp Med. 2006;6(4):150-156.
27. Middleton E Jr, Drzewiecki G, Krishnarao D. Quercetin: an inhibitor of antigen-induced human basophil histamine release. J Immunol. 1981;127(2):546-550.

28. Middleton E Jr, Drzewiecki G. Flavonoid inhibition of human basophil histamine release stimulated by various agents. Biochem Pharmacol. 1984;33(21):3333-3338.

29. Togni S, Maramaldi G, Meneghin M, et al. Antiallergic and antioxidant properties of a lecithin-based delivery form of quercetin: an in vitro evaluation. Esperienze Dermatologiche. 2016; in press.

\section{Publish your work in this journal}

Clinical, Cosmetic and Investigational Dermatology is an international, peer-reviewed, open access, online journal that focuses on the latest clinical and experimental research in all aspects of skin disease and cosmetic interventions. All areas of dermatology will be covered; contributions will be welcomed from all clinicians and basic science researchers globally. This journal is indexed on CAS The manuscript management system is completely online and includes a very quick and fair peer-review system, which is all easy to use. Visit http://www.dovepress.com/testimonials.php to read real quotes from published authors.

Submit your manuscript here: http://www.dovepress.com/clinical-cosmetic-and-investigational-dermatology-journal 